\title{
Novel 3-Scroll Chua's Attractor with One Saddle-Focus and Two Stable Node-Foci
}

\author{
Kaibin Chu, Zhengwei Zhu $\mathbb{D}$, Hui Qian, and Huagan Wu $\mathbb{D}$ \\ School of Information Science and Engineering, Changzhou University, Changzhou 213164, China \\ Correspondence should be addressed to Zhengwei Zhu; zhuzw888@126.com
}

Received 23 October 2017; Revised 15 December 2017; Accepted 3 January 2018; Published 31 January 2018

Academic Editor: Marco Spadini

Copyright (C) 2018 Kaibin Chu et al. This is an open access article distributed under the Creative Commons Attribution License, which permits unrestricted use, distribution, and reproduction in any medium, provided the original work is properly cited.

With new three-segment piecewise-linearity in the classic Chua's system, two new types of 2-scroll and 3-scroll Chua's attractors are found in this paper. By changing the outer segment slope of the three-segment piecewise-linearity as positive, the new 2-scroll Chua's attractor has emerged from one zero index-1 saddle-focus and two symmetric stable nonzero node-foci. In particular, by newly introducing a piecewise-linear control function, an improved Chua's system only with one zero index- 2 saddle-focus and two stable nonzero node-foci is constructed, from which a 3-scroll Chua's attractor is converged. Some remarks for Chua's nonlinearities and the generating chaotic attractors are discussed, and the stabilities at the three equilibrium points are then analyzed, upon which the emerging mechanisms of the novel 2-scroll and 3-scroll Chua's attractors are explored in depth. Furthermore, an analog electronic circuit built with operational amplifier and analog multiplier is designed and hardware circuit experiments are measured to verify the numerical simulations. These novel 2-scroll and 3-scroll Chua's attractors reported in this paper are completely different from the classic Chua's attractors, which will enrich the dynamics of the classic Chua's system.

\section{Introduction}

As it has been shown, Chua's circuit is a relatively simple circuit which has rapidly become a paradigm for chaos [1]. In the past three decades, numerous works have been reported on this circuit, including realization schemes, experimental measurements, numerical observations, and theoretical proofs [2-7]. The classic Chua's system with a simple algebraic structure is a dimensionless form of Chua's equations, and its nonlinearity formed by Chua's diode, called Chua's nonlinearity in this paper, is three-segment piecewise-linear. Consequently, the classic Chua's system has three unstable equilibrium points, one zero index-1 saddle-focus and two symmetric nonzero index-2 saddle-foci, resulting in the generation of a self-excited 2-scroll chaotic attractor [1].

By modifying Chua's nonlinearity with multiple-segment piecewise-linear or continuous nonlinear functions, Chua's system can be generalized to a system exhibiting more complex attractors [8-10], that is, self-excited multiscroll Chua's chaotic attractors. Generally, self-excited multiscroll or multiwing chaotic attractors are generated by disposing unstable index-2 saddle-foci in terms of added breakpoints in the model system [11, 12], which shows great theoretical and practical significance due to the applications to encrypted communication, chaos synchronization, and some other fields of Chua's systems with multiscroll chaotic attractors [13].

Chaotic dynamics has been investigated in numerous fields of science and engineering including mathematics, physics, chemistry, electronic circuit, information, and mechanical engineering [14-21]. In the past few years, a new type of attractor, defined as hidden attractor, has been found in large numbers of nonlinear dynamical systems [19-25]. Different from self-excited attractor, hidden attractor, whose attraction basin does not intersect with small neighborhoods of the equilibria of the system [19-21], is sensitive to the initial conditions and special analytical-numerical procedure should be adopted to locate its attractive basin [20]. Recently, by developing this procedure, hidden chaotic spiral attractor has been numerically observed in the classic Chua's system with one stable zero equilibrium point [20,26]. Furthermore, two coexisting hidden attractors have been numerically revealed and a dynamical route from one hidden limit cycle to hidden twin chaotic attractors by period-doubling 


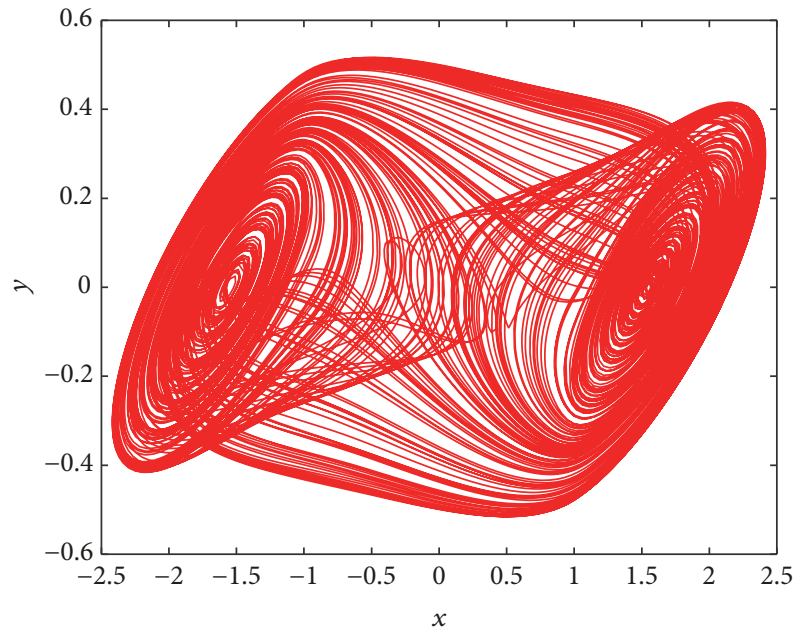

(a)

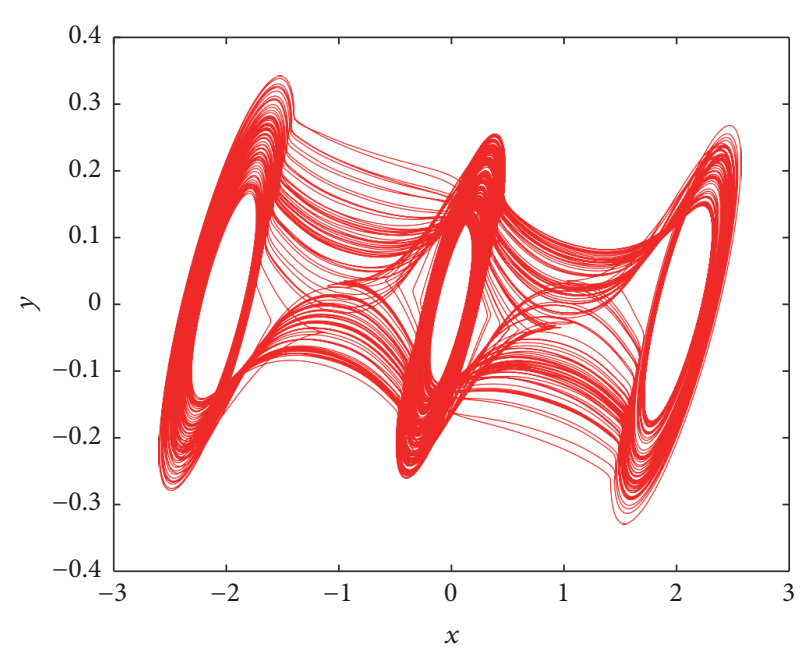

(b)

Figure 1: Two self-excited Chua's chaotic attractors with two and three index-2 saddle-foci. (a) Two-scroll with two nonzero index-2 saddlefoci; (b) 3-scroll with three index-2 saddle-foci.

bifurcations has been reported in the classic Chua's system $[26,27]$.

The mechanism to have different types of attractors in the classic Chua's system is due to different Chua's nonlinearities [1, 26-28]. Different Chua's nonlinearities formulate the three equilibrium points with different characteristics, leading to the generations of different types of Chua's attractors. This paper finds 2-scroll and 3-scroll Chua's attractors in the classic Chua's system by setting the outer segment slope of threesegment piecewise-linearity to be positive and introducing a new piecewise- linear control function $[10,28]$. A distinct characteristic is that the scroll number of the new Chua's attractor does not associate with the number of index-2 saddle-foci of the classic Chua's system. To the best of our knowledge, there are no such reports in public literature. These findings will further enrich the dynamics of the classic Chua's system [1].

This rest of the paper is organized as follows. Section 2 discusses two kinds of Chua's nonlinearities and the correspondingly generating Chua's attractors and introduces a new three-segment piecewise-linearity. Section 3 studies a 2-scroll Chua's attractor with one zero index-1 saddle-focus and two symmetric stable nonzero node-foci. Section 4 investigates a 3 -scroll Chua's attractor only with one zero index-2 saddlefocus and two symmetric stable nonzero node-foci by newly introducing a piecewise-linear control function. Section 5 conducts hardware circuit experiments to confirm the theoretical analyses. The conclusions are summarized in the last section.

\section{Remarks for Chua's Nonlinearities and the Generating Chaotic Attractors}

The classic Chua's system is described by the following dimensionless equations [1]:

$$
\dot{x}=\alpha(y-x)-\alpha f(x),
$$

$$
\begin{aligned}
& \dot{y}=x-y+z, \\
& \dot{z}=-\beta y-\gamma z,
\end{aligned}
$$

where $\alpha, \beta$, and $\gamma$ are constant parameters and

$$
f(x)=b x+0.5(a-b)(|x+1|-|x-1|)
$$

is used for describing the three-segment piecewise-linear characteristic of system (1), with $a, b$ being the slopes of the inner and outer segments of $f(x)$. Note that the parameter $\gamma$ is usually ignored; that is, $\gamma=0$.

When the parameters are given as $\alpha=10, \beta=14.5140$, $\gamma=0, a=-1.1970$, and $b=-0.6464$ and the initial conditions are selected as $(0.001,0,0)$, the phase portrait of the classical 2-scroll chaotic attractor has emerged from two symmetric index-2 saddle-foci, as shown in Figure 1(a). Note that there exist the relations of $a<0, b<0,|a|>1$, and $|b|<1$.

In [10], through replacing the characteristic function (2) by a new piecewise-linear function (in the $x$ and $z$ variables) with a controllable threshold value $\mu$ in the $z$-axis, a 3-scroll chaotic attractor with three index-2 saddle-foci is converged, as shown in Figure 1(b), where $\alpha=10.0, \beta=14.0, \gamma=0, a=$ $-1.43, b=-0.59$, and $\mu=1$. The mechanism for generating the 3 -scroll chaotic attractor is that the zero index-1 saddlefocus of the classic Chua's system is changed as a zero index-2 saddle-focus by modifying the nonlinearity of (2), leading to the formation of an additional index-2 saddle-focus.

However, it is found that a hidden Chua's spiral attractor with one stable zero node-focus exists when the parameters $\alpha=8.8, \beta=12.0732, \gamma=0.0052, a=-0.1768$, and $b=-1.1468$, and the initial conditions are $(9.4287,-0.5945$, and -13.4705) [20,21], as shown in Figure 2(a), whereas when the parameters are utilized as $\alpha=8.4562, \beta=12.0732$, $\gamma=0.0052, a=-0.1768$, and $b=-1.1468$, and the initial conditions are set as $(-6.0489,0.0839$, and 8.7739) and $(6.0489,-0.0839$, and -8.7739$)$, respectively, hidden twin 


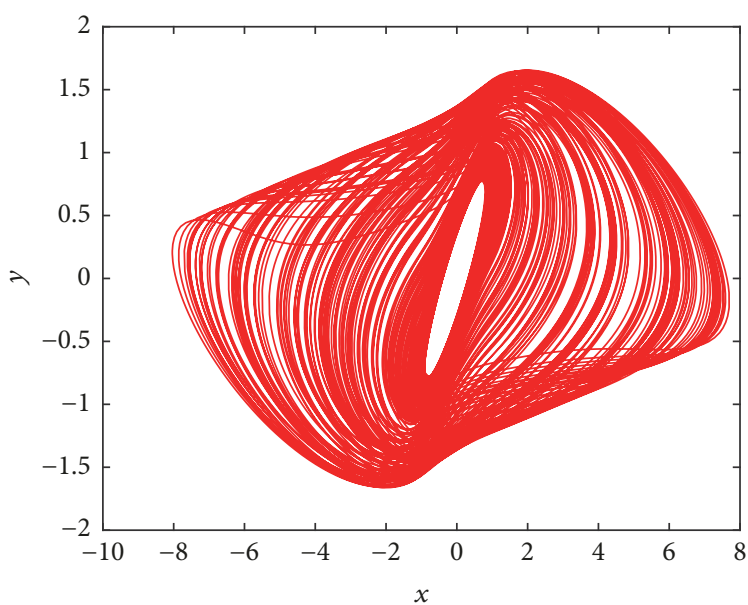

(a)

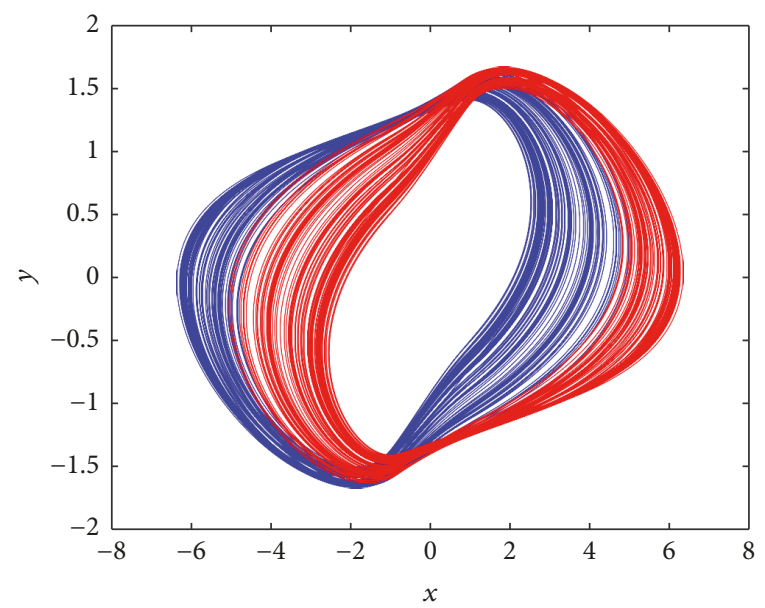

(b)

FIGURE 2: Hidden Chua's chaotic attractors with one stable zero node-focus. (a) Hidden Chua's spiral attractor; (b) hidden twin Chua's spiral attractors, where the initial conditions for hidden attractors in blue and red colors are $(-6.0489,0.0839$, and 8.7739$)$ and $(6.0489,-0.0839$, and -8.7739 ), respectively.

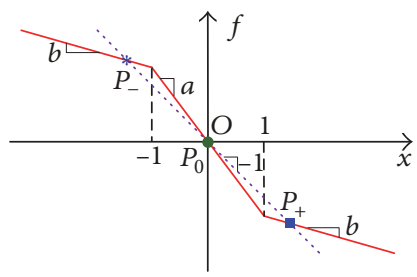

(a)

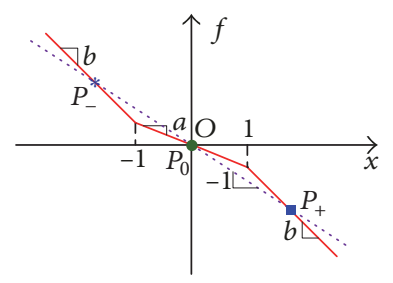

(b)

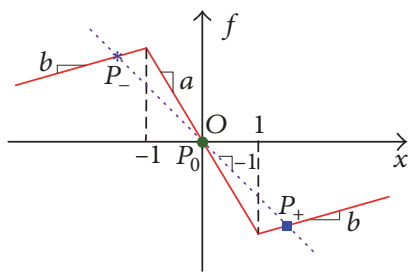

(c)

FIGURE 3: Three kinds of three-segment piecewise-linearity with different relations of the slopes of the inner and outer segments $a$ and $b$. (a) The relations of $a<0, b<0,|a|>1$, and $|b|<1$; (b) the relations of $a<0, b<0,|a|<1$, and $|b|>1$; (c) the relations of $a<0, b>0$, and $|a|>1$.

Chua's spiral attractors with one stable zero node-focus are observed [26, 27], as shown in Figure 2(b). Note that Chua's nonlinearity is modified for the generations of these hidden Chua's attractors, and there exist the relations of $a<0, b<0$, $|a|<1$, and $|b|>1$ and Chua's nonlinearity is different from that in Figure 1(a).

Chua's nonlinearity $f(x)$ of (2) is featured by two parameters $a, b$, whose relations formulate the different Chua's attractors. For the classic Chua's system, the original threesegment piecewise- linearity is depicted in Figure 3(a), where the conditions of $a<0, b<0,|a|>1$, and $|b|<1$ are satisfied for generating a self-excited Chua's attractor, as shown in Figure 1(a), resulting in the emergence of one zero index1 saddle-focus and two symmetric nonzero index- 2 saddlefoci [1]. Nevertheless, a modified three-segment piecewiselinearity is plotted in Figure 3(b), where the relations of $a<0$, $b<0,|a|<1$, and $|b|>1$ are satisfied for generating a hidden Chua's attractor, as shown in Figure 2, leading to the formation of one stable zero node-focus and two nonzero index-1 saddle-focus [20].

If the outer segment slope of the three-segment piecewise-linearity $f(x)$ is changed as positive, a new Chua's nonlinearity is presented [28], as shown in Figure 3(c), where the relations are built as $a<0, b>0$, and $|a|>1$, and the characteristics of three equilibrium points are altered, which lead to the occurrence of a new type of Chua's chaotic attractor as discussed in the following sections.

It is concluded that Chua's system (1) has the same three equilibrium points, one zero equilibrium point $P_{0}$ and two symmetric nonzero equilibrium points $P_{ \pm}$, as shown in Figure 3. With different Chua's nonlinearities, these equilibrium points have different dynamical characteristics, from which different types of Chua's chaotic attractors have emerged.

\section{2-Scroll Chua's Attractor with Two Stable Node-Foci}

In our next work, system (1) with Chua's nonlinearity characterized by Figure 3(c) is considered, which is defined as a modified Chua's system. It should be illustrated that, with $a<0, b>0$, and $|a|>1$, the parameter region of interest corresponds to the first quadrant of Chua's parameter space $(\alpha>0, \beta>0$, and $\gamma=0)$ in the considered region.

Three same equilibrium points of Chua's system (1) are given by

$$
\begin{aligned}
& P_{0}=(0,0,0), \\
& P_{ \pm}=( \pm k, 0, \mp k),
\end{aligned}
$$




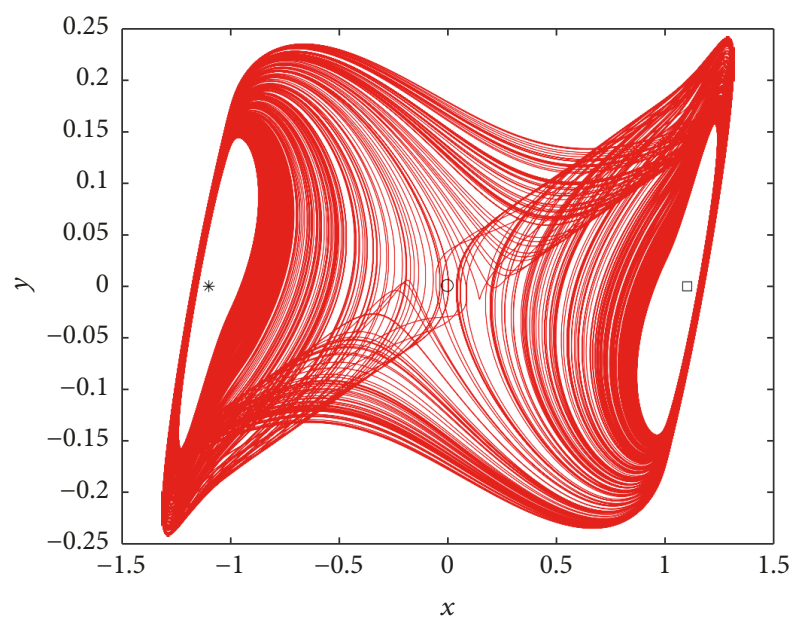

(a)

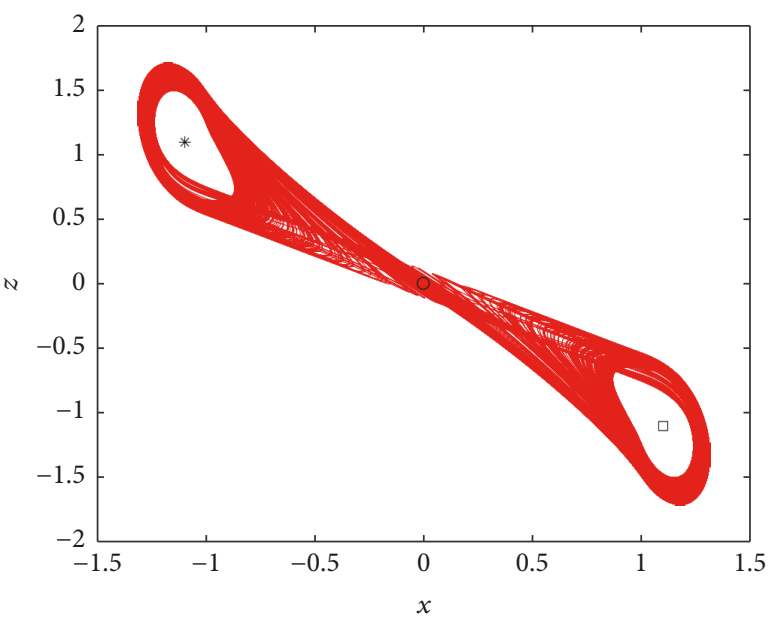

(b)

FIGURE 4: 2-scroll Chua's chaotic attractor with two symmetric stable nonzero node-foci. (a) Phase portrait in the $x$ - $y$ plane; (b) phase portrait in the $x-z$ plane.

where $k=(b-a) /(1+b)$. The Jacobian matrix at the equilibrium point is given as

$$
\mathbf{J}(c)=\left[\begin{array}{ccc}
-\alpha(c+1) & \alpha & 0 \\
1 & -1 & 1 \\
0 & -\beta & 0
\end{array}\right],
$$

where $c=a$ for $P_{0}$ and $c=b$ for $P_{ \pm}$. The characteristic equation is

$$
P(\lambda)=\lambda^{3}+a_{1} \lambda^{2}+a_{2} \lambda+a_{3}=0,
$$

where $a_{1}=\alpha(c+1)+1, a_{2}=\alpha c+\beta$, and $a_{3}=\alpha \beta(c+1)$. The Routh-Hurwitz conditions for the above cubic polynomial are given by

$$
\begin{aligned}
a_{1} & =\alpha(c+1)+1>0, \\
a_{3} & =\alpha \beta(c+1)>0, \\
a_{1} a_{2}-a_{3} & =\alpha c[\alpha(c+1)+1]+\beta>0 .
\end{aligned}
$$

Note that for the modified Chua's system (1) with Chua's nonlinearity of Figure 3(c), there exist the relations of $a<0$, $b>0$, and $|a|>1$. For $P_{0}, c=a$. In view of $a+1<0$, the second condition, that is, $a_{3}=\alpha \beta(a+1)>0$, is not satisfied. Thus, $P_{0}$ is always unstable, whereas for $P_{ \pm}, c=b$. Due to $b>0$, the three conditions of (6) are satisfied, implying that $P_{ \pm}$are always stable, no matter the positive values of $\alpha$ and $\beta$.

Considering that the system parameters $\alpha=5.8, \beta=$ $6.2, a=-1.1$, and $b=0.02$ and the initial conditions $(0.001,0,0)$, the phase portraits of a 2 -scroll Chua's chaotic attractor in two different planes are plotted in Figures 4(a) and 4(b), respectively, where the mark $\bigcirc$ stands for the zero equilibrium point, and the marks $\square$ and $*$ represent the two symmetric nonzero equilibrium points. Correspondingly, the eigenvalues at these three equilibrium points of (3) are calculated as

$$
\begin{array}{ll}
P_{0}: & \lambda_{1}=1.4398 \\
& \lambda_{2,3}=-0.9299 \pm j 1.2779, \\
P_{ \pm}: & \lambda_{1,2}=-0.0669 \pm j 2.3246, \\
& \lambda_{3}=-6.7821 .
\end{array}
$$

It means that $P_{0}$ is a saddle-focus having a positive real root and two complex conjugate roots with negative real parts, that is, index-1 saddle-focus, whereas $P_{ \pm}$are two symmetric stable node-foci having two complex conjugate roots with negative real parts and a negative real root.

Consequently, when the two parameters $a$ and $b$ of the three-segment piecewise-linearity in (2) satisfy the conditions

$$
\begin{gathered}
a<0, \\
b>0, \\
|a|>1
\end{gathered}
$$

the modified Chua's system (1) has two symmetric stable nonzero node-foci but can generate 2-scroll Chua's chaotic attractor. This type of attractor is completely different from the self-excited 2-scroll chaotic attractor reported in [1] and also different from the hidden chaotic spiral attractor reported in $[20,26]$.

\section{3-Scroll Chua's Attractor with One Saddle-Focus and Two Stable Node-Foci}

Reconsider the modified Chua's system (1) with Chua's nonlinearity characterized by Figure 3(c). Usually, by defining 


$$
\begin{aligned}
h(x) & \triangleq x+f(x) \\
& =(b+1) x+0.5(a-b)(|x+1|-|x-1|)
\end{aligned}
$$

an equivalent form of the modified Chua's system (1) is then expressed as

$$
\begin{aligned}
& \dot{x}=\alpha y-\alpha h(x), \\
& \dot{y}=x-y+z, \\
& \dot{z}=-\beta y .
\end{aligned}
$$

Likewise, the nonlinearity $h(x)$ is also a three-segment piecewise-linearity.

With (7a), the zero equilibrium point $P_{0}$ of system (10) is an index- 1 saddle-focus. In order to make $P_{0}$ convert into an index-2 saddle-focus, a piecewise-linear control function with two variables $x$ and $z$ is introduced, which can be expressed by [10]

$$
\begin{aligned}
h_{\mu}(x, z) & =h(x)[1+\operatorname{sgn}(z-\mu)-\operatorname{sgn}(z+\mu)] \\
& = \begin{cases}h(x), & |z| \geq \mu, \\
-h(x), & |z| \leq \mu,\end{cases}
\end{aligned}
$$

where the parameter $\mu$ is a controllable threshold value in the $z$-axis and satisfies $0<\mu<1$. Thus, by replacing $h(x)$ with $h_{\mu}(x, z)$, an improved Chua's system is obtained as

$$
\begin{aligned}
& \dot{x}=\alpha y-\alpha h_{\mu}(x, z), \\
& \dot{y}=x-y+z, \\
& \dot{z}=-\beta y,
\end{aligned}
$$

where $\alpha>0$ and $\beta>0$.

Similarly, the improved Chua's system described by (12) has three equilibrium points given in (3). Considering the piecewise-linear control function $h_{\mu}(x, z)$ with the condition $0<\mu<1$, the zero equilibrium point $P_{0}$ is situated in the inner region of the $x-z$ plane and the two symmetric nonzero equilibrium points $P_{ \pm}$are located in the outer regions of the $x-z$ plane. Thus, the stability of $P_{0}$ is forcedly altered and the stabilities of $P_{ \pm}$are still maintained unchanged.

Therefore, the Jacobian matrix evaluated at the zero equilibrium $P_{0}$ is derived as

$$
\mathbf{J}\left(P_{0}\right)=\left[\begin{array}{ccc}
\alpha(a+1) & \alpha & 0 \\
1 & -1 & 1 \\
0 & -\beta & 0
\end{array}\right]
$$

The characteristic equation at the zero equilibrium point $P_{0}$ is yielded as

$$
P(\lambda)=\lambda^{3}+b_{1} \lambda^{2}+b_{2} \lambda+b_{3}=0,
$$

where $b_{1}=1-\alpha(a+1), b_{2}=\beta-\alpha(a+2)$, and $b_{3}=-\alpha \beta(a+1)$. The coefficients of (14) are all nonzero and the Routh-Hurwitz conditions are given by

$$
\begin{aligned}
b_{1} & =1-\alpha(a+1)>0, \\
b_{3} & =-\alpha \beta(a+1)>0, \\
b_{1} b_{2}-b_{3} & =\alpha(a+2)(\alpha a+\alpha-1)+\beta>0 .
\end{aligned}
$$

When $a$ and $b$ of $h(x)$ satisfy the conditions given in (8), there exists $a+1<0$. Thus, the first two conditions, that is, $b_{1}>0$ and $b_{3}>0$, are satisfied, but the third condition, that is, $b_{1} b_{2}-$ $b_{3}>0$, cannot be satisfied when $\beta<-\alpha(a+2)(\alpha a+\alpha-1)$. For example, the system parameters of the improved Chua's system are chosen as

$$
\begin{aligned}
& \alpha=5.8, \\
& \beta=5.8, \\
& a=-1.1, \\
& b=0.02, \\
& \mu=0.4 .
\end{aligned}
$$

The third condition of (15) cannot be satisfied, implying that $P_{0}$ is unstable.

In contrast, the Jacobian matrix evaluated at the two symmetric nonzero equilibria $P_{ \pm}$is obtained as

$$
\mathbf{J}\left(P_{ \pm}\right)=\left[\begin{array}{ccc}
-\alpha(b+1) & \alpha & 0 \\
1 & -1 & 1 \\
0 & -\beta & 0
\end{array}\right]
$$

which is identical with the Jacobian matrix (4) for the case of $P_{ \pm}$. Hence, $P_{ \pm}$are always stable.

For the system parameters given in (16), the eigenvalues at three equilibrium points are calculated as

$$
\begin{aligned}
P_{0}: & \lambda_{1,2}=0.2496 \pm j 1.2472, \\
& \lambda_{3}=-2.0792, \\
P_{ \pm}: & \lambda_{1,2}=-0.0635 \pm j 2.2472, \\
& \lambda_{3}=-6.7891 .
\end{aligned}
$$

It means that $P_{0}$ is an index- 2 saddle-focus having two complex conjugate roots with positive real parts and a negative real root, whereas $P_{ \pm}$are two stable node-foci having two complex conjugate roots with negative real parts and a negative real root.

For the system parameters given in (16) and the initial conditions $(0.001,0,0)$, the phase portraits of the chaotic attractor in three different planes are plotted in Figures 5(a), 5(b), and 5(c), respectively, and Poincaré mapping on the $y=0$ section is depicted in Figure 5(d). It can be observed from Figure 5 that a novel 3-scroll Chua's chaotic attractor is generated in the improved Chua's system.

It should be clarified that the newly found 3-scroll Chua's chaotic attractor has one zero index- 2 saddle-focus 


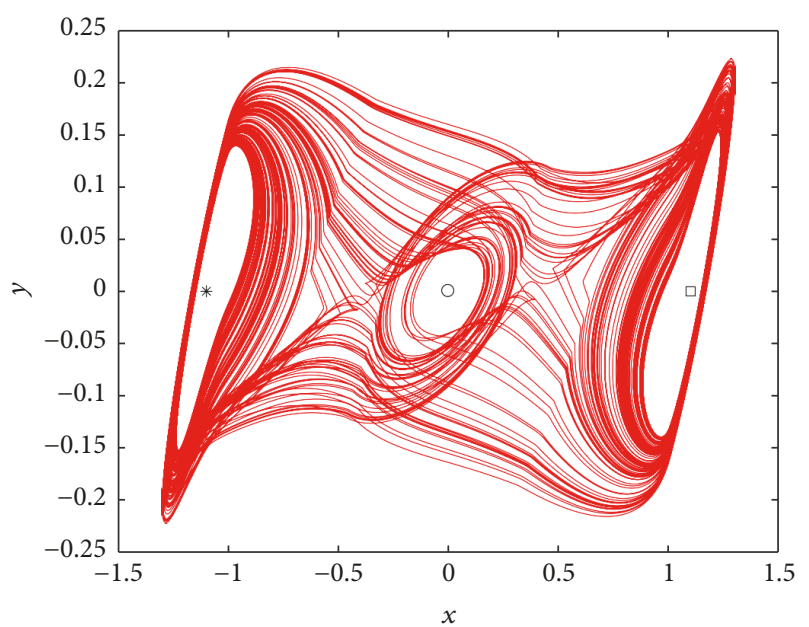

(a)

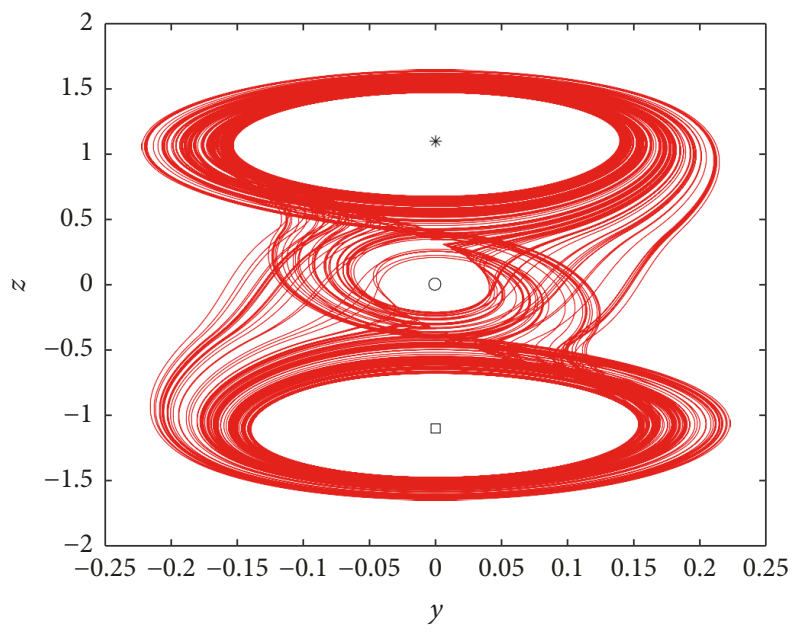

(c)

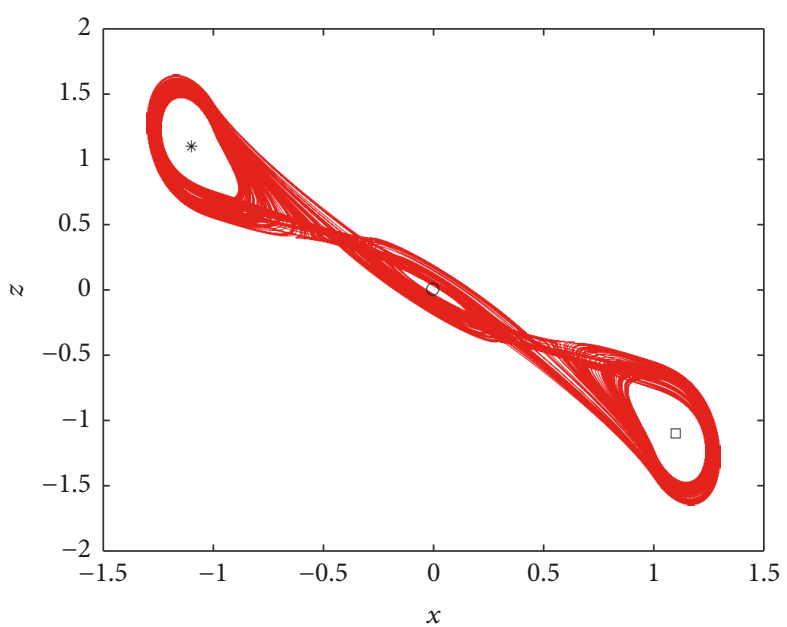

(b)

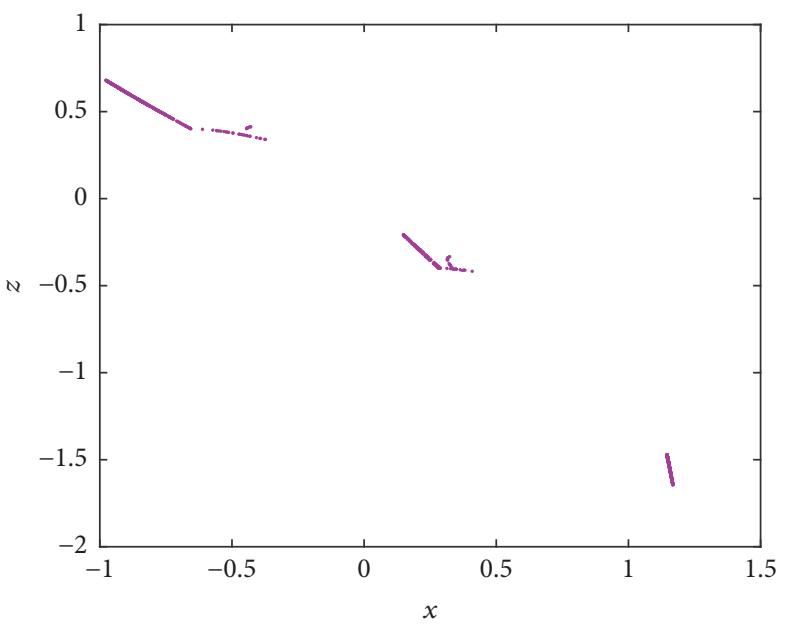

(d)

Figure 5: Novel 3-scroll Chua's chaotic attractor with one zero index-2 saddle-focus and two stable nonzero node-foci. (a) Phase portrait in the $x-y$ plane; (b) phase portrait in the $x-z$ plane; (c) phase portrait in the $y-z$ plane; (d) Poincaré mapping in the $x-z$ plane.

and two stable nonzero node-foci, whereas the 3-scroll attractor reported in [10] has three index-2 saddle-foci. Therefore, the new 3-scroll Chua's chaotic attractor is different from the self-excited or hidden Chua's chaotic attractors [19-21].

Additionally, the system parameters given in (16) and other initial conditions are considered. When the initial conditions $(1,0,0)$ are assigned, the improved Chua's system exhibits a chaotic spiral attractor, as shown in Figure 6(a), while when the initial conditions $(-1,0,1)$ and $(1,0,-1)$ are selected, respectively, the improved Chua's system displays two stable point attractors settling down the two symmetric nonzero equilibrium points $P_{ \pm}$[29], as shown in Figure 6(b), where the trajectories from the initial states of $(-1,0,1)$ are colored in blue and those from $(1,0,-1)$ are colored in red. The above results illustrate that just like the dynamical systems reported in [29-35], the improved Chua's system can present multistability.

\section{Circuit Realizations and Hardware Experiments}

An analog electronic circuit built with operational amplifier and analog multiplier is designed to physically realize the improved Chua's system for generating the novel 3-scroll Chua's chaotic attractor, as shown in Figure 7 [9, 30, 31]. The designed circuit is constructed by three parts: the first part is a basic three-dimensional Chua's system with three integration channels, as shown in Figure 7(a); the second part is the circuit for realizing the piecewise-linearity $h(x)$, as shown in Figure $7(b)$; and the third part is the circuit for implementing the control function $h_{\mu}(x, z)$, as shown in Figure 7(c).

In Figure 7, the system parameters given in (16) are used, and the time constant $R_{0} C_{0}$ of the integrator is determined by $R_{0}=10 \mathrm{k} \Omega$ and $C_{0}=33 \mathrm{nF}$. The operational amplifiers OP07CP and analog multipliers AD633JNZ with $\pm 15 \mathrm{~V}$ power supplies are utilized. Thus, the saturation voltage of the 


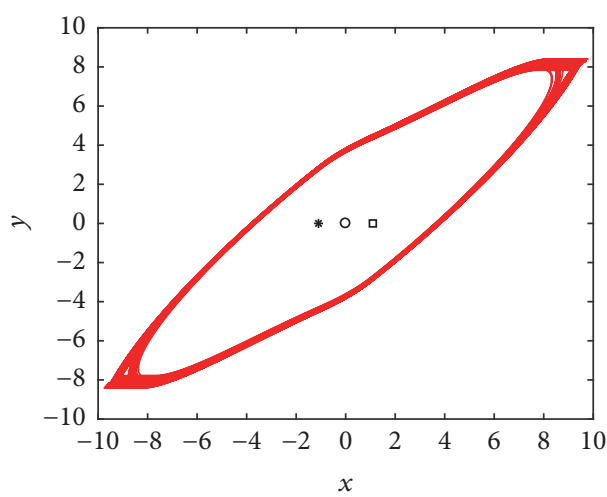

(a)

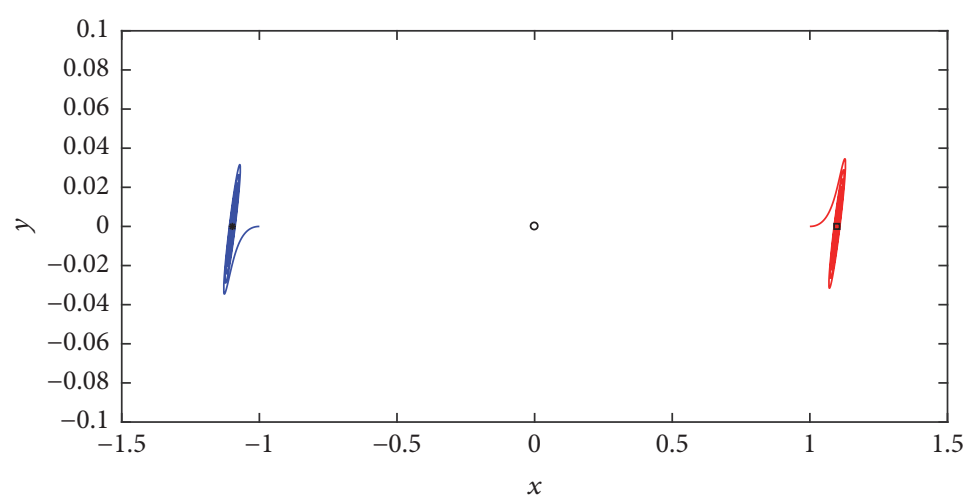

(b)

FIGURE 6: Multistability in the improved Chua's system, where the phase portraits are in the $x-y$ plane. (a) Chaotic spiral attractor for $(1,0$, $0)$; (b) two stable point attractors for $(-1,0,1)$ and $(1,0,-1)$.

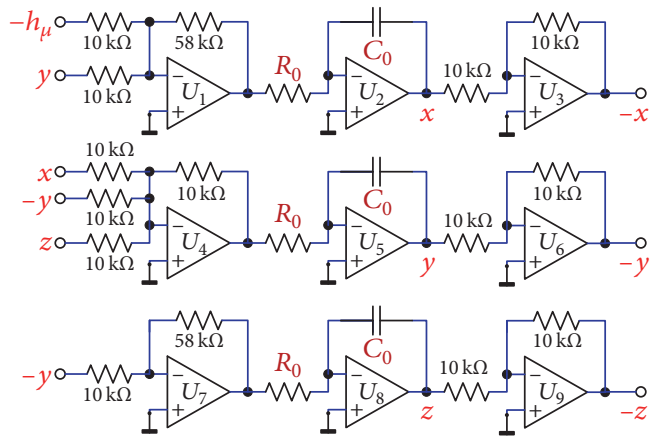

(a)

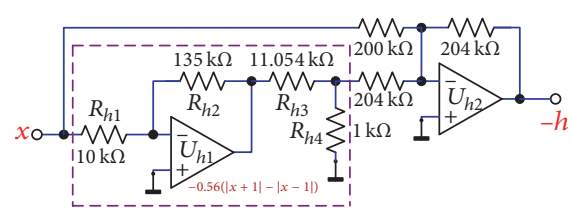

(b)

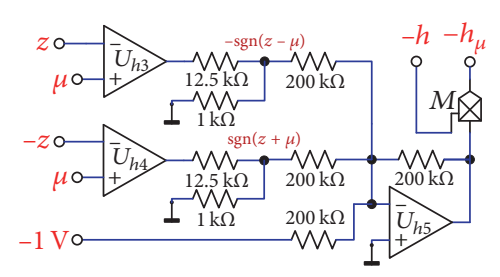

(c)

Figure 7: The improved Chua's chaotic system. (a) Basic circuit with three integration channels; (b) $h(x)$ piecewise-linearity realization; (c) $h_{\mu}(x, z)$ control function realization.

operational amplifier output is $E_{\text {sat }}=13.5 \mathrm{~V}$. Note that in Figure $7(\mathrm{~b})$ the following relations should be satisfied as

$$
\begin{aligned}
& R_{h 2}=E_{\mathrm{sat}} R_{h 1}=135 \mathrm{k} \Omega, \\
& R_{h 3}=\frac{R_{h 2} R_{h 4}}{(b-a) R_{h 1}}-R_{h 4}=11.0536 \mathrm{k} \Omega .
\end{aligned}
$$

The experimental prototype of the improved Chua's chaotic system is photographed in Figure 8. By Tektronix TDS 3034C digital oscilloscope, the phase portraits of novel 3 -scroll Chua's chaotic attractor in three different planes are experimentally measured, as shown in Figures 9(a), 9(b), and 9(c), respectively. Furthermore, if the feedback resistance up the op-amp $U_{7}$ in Figure $7(\mathrm{a})$ is changed as $62 \mathrm{k} \Omega$ and the realization circuit of $h(x)$ is directly linked to the input terminal of the op-amp $U_{1}$ in Figure 7(a), the circuit realization of the modified Chua's system for generating 2-scroll Chua's chaotic attractor is established, from which the phase portraits of 2-scroll Chua's chaotic attractor in two different planes are experimentally captured, as plotted in Figures 10(a) and 10(b), respectively. Obviously, the experimental measurements of Figures 9 and 10 are consistent with the numerical simulations of Figures 5 and 4, respectively.

\section{Conclusion}

In this paper, by changing Chua's nonlinearity and introducing piecewise-linear control function, a new type of 2scroll and 3-scroll Chua's chaotic attractors generated from an improved Chua's system are studied through theoretical analyses, numerical simulations, and hardware circuit experiments. The traditional 2-scroll and 3-scroll Chua's chaotic 


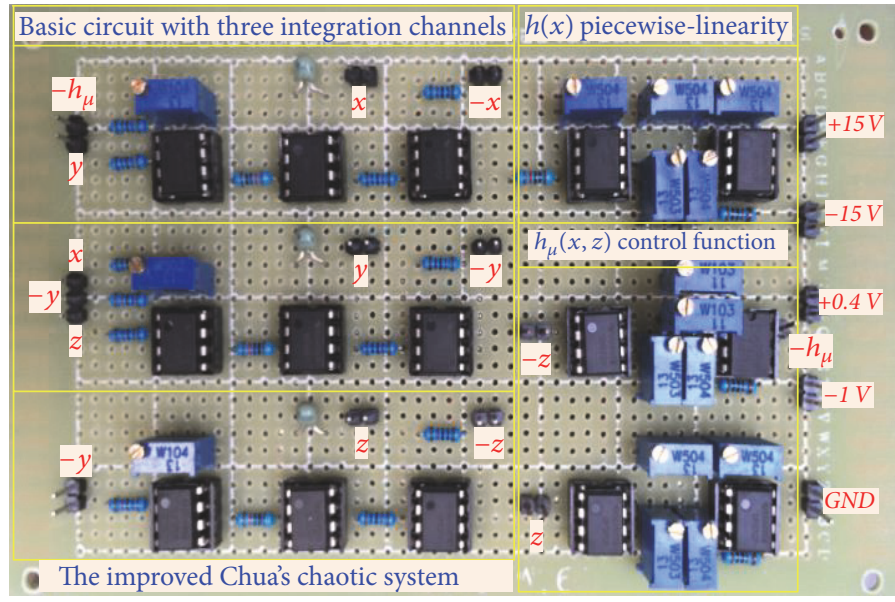

FIGURE 8: Photograph of the experimental prototype, the left part is basic circuit with three integration channels, whereas the right part is $h(x)$ piecewise-linearity circuit and $h_{\mu}(x, z)$ control function circuit.

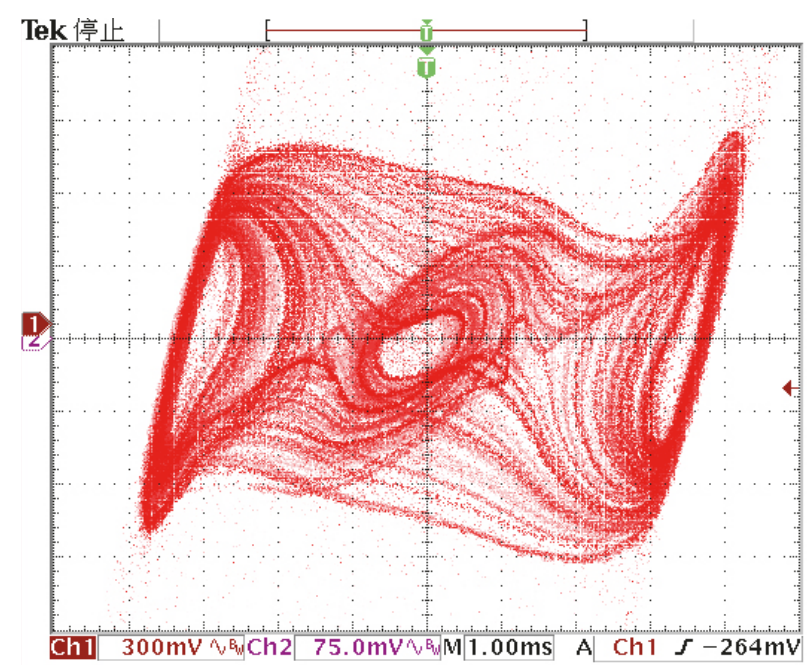

(a)

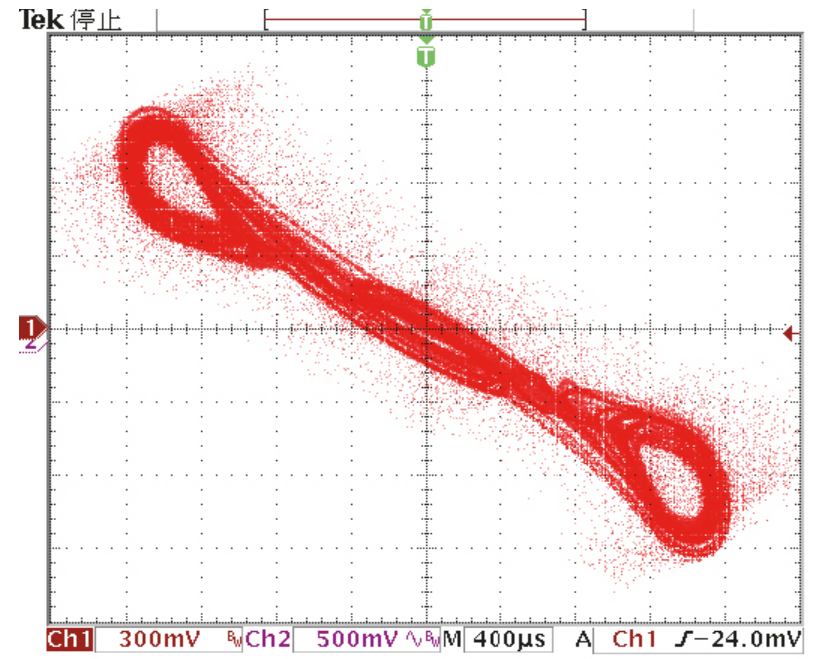

(b)

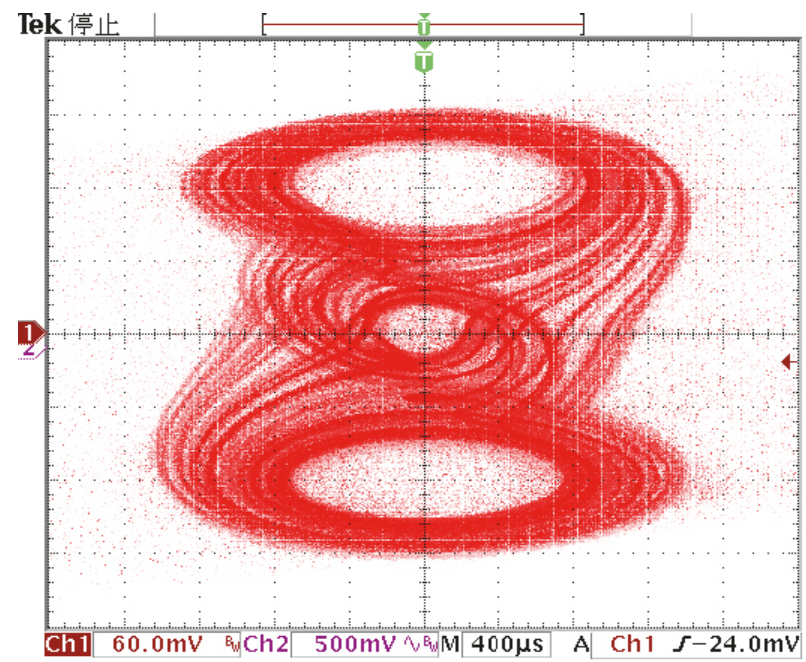

(c)

FIGURE 9: Experimentally measured phase portraits of 3-scroll Chua's attractor. (a) Phase portrait in the $x$ - $y$ plane; (b) phase portrait in the $x$ - $z$ plane; (c) phase portrait in the $y$ - $z$ plane. 


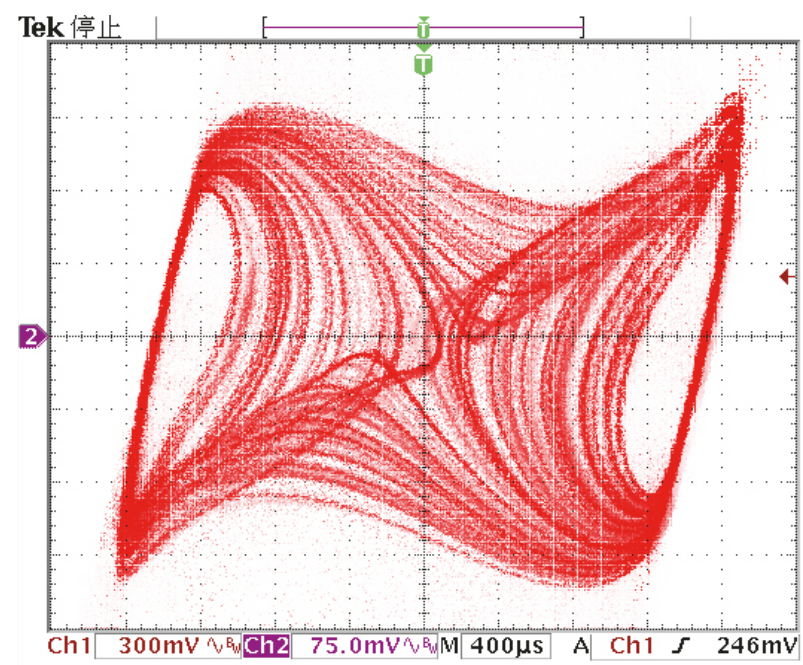

(a)

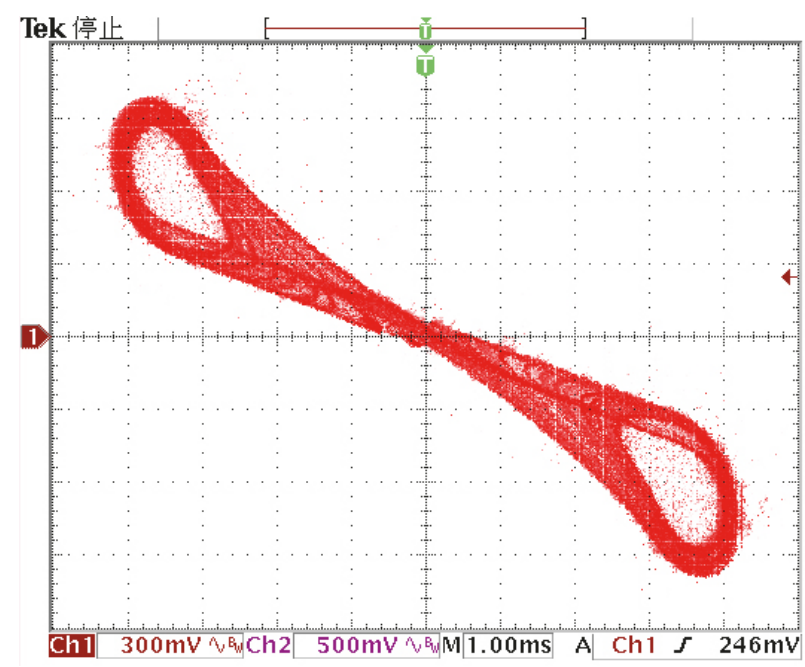

(b)

FIGURE 10: Experimentally measured phase portraits of 2-scroll Chua's attractor. (a) Phase portrait in the $x$ - $y$ plane; (b) phase portrait in the $x-z$ plane.

attractors are all excited from index-2 saddle-foci, whereas the proposed 2-scroll and 3-scroll Chua's chaotic attractors have emerged from two symmetric stable node-foci or one index-2 saddle-focus and two symmetric stable node-foci. Therefore, it is very interesting and particularly valuable to discover this 3-scroll chaotic attractor in the improved Chua's system only with one zero index- 2 saddle-focus and two symmetric stable nonzero node-foci. Due to this, the proposed 3-scroll Chua's chaotic system may be regarded as a special paradigm for investigating special nonlinear phenomena different from self-excited and hidden Chua's chaotic attractors.

\section{Conflicts of Interest}

The authors declare that they have no conflicts of interest.

\section{Acknowledgments}

This work was supported by the National Natural Science Foundations of China under Grants nos. 51777016, 61772090, and 51607013 .

\section{References}

[1] L. Fortuna, M. Frasca, and M. G. Xibilia, "Chuas circuit implementations: yesterday, today and tomorrow," World Scientific, 2009.

[2] L. O. CHUA, “Chua’s circuit: an overview ten years later," Journal of Circuits, Systems and Computers, vol. 04, no. 02, pp. 117-159, 1994.

[3] M. P. Kennedy, "Robust OP Amp Realization of Chua's Circuit," Frequenz, vol. 46, no. 3-4, pp. 66-80, 1992.

[4] L. Pivka and V. Špány, "Boundary surfaces and basin bifurcations in Chua's circuit," Journal of Circuits, Systems and Computers, vol. 3, no. 2, pp. 441-470, 1993.
[5] R. O. Medrano-T and R. Rocha, "The negative side of Chua's circuit parameter space: Stability analysis, period-adding, basin of attraction metamorphoses, and experimental investigation," International Journal of Bifurcation and Chaos, vol. 24, no. 9, Article ID 1430025-1, 2014.

[6] C. K. Volos, I. M. Kyprianidis, and I. N. Stouboulos, "An universal phenomenon in mutually coupled chua's circuit family," Journal of Circuits, Systems and Computers, vol. 23, no. 2, Article ID 1450028, 2014.

[7] B. Bao, N. Wang, M. Chen, Q. Xu, and J. Wang, "Inductorfree simplified Chua's circuit only using two-op-amp-based realization," Nonlinear Dynamics, vol. 84, no. 2, pp. 511-525, 2016.

[8] M. E. Yalçin, J. A. K. Suykens, and J. Vandewalle, "Experimental confirmation of 3- and 5-scroll attractors from a generalized Chua's circuit," IEEE Transactions on Circuits and Systems I: Fundamental Theory and Applications, vol. 47, no. 3, pp. 425429, 2000.

[9] S. Yu, W. K. S. Tang, and G. Chen, "Generation of $\mathrm{n} \times \mathrm{m}-$ scroll attractors under a Chua-circuit framework," International Journal of Bifurcation and Chaos, vol. 17, no. 11, pp. 3951-3964, 2007.

[10] Z. Elhadj and J. C. Sprott, "Generating 3-scroll attractors from one Chua circuit," International Journal of Bifurcation and Chaos, vol. 20, no. 1, pp. 135-144, 2010.

[11] D. Chen, Z. Sun, X. Ma, and L. Chen, "Circuit implementation and model of a new multi-scroll chaotic system," International Journal of Circuit Theory and Applications, vol. 42, no. 4, pp. 407-424, 2014.

[12] S. Yu, J. Lu, X. Yu, and G. Chen, "Design and implementation of grid multiwing hyperchaotic Lorenz system family via switching control and constructing super-heteroclinic loops," IEEE Transactions on Circuits and Systems I: Regular Papers, vol. 59, no. 5, pp. 1015-1028, 2012.

[13] L. Gámez-Guzmán, C. Cruz-Hernández, R. M. López-Gutiérrez, and E. E. García-Guerrero, "Synchronization of Chua’s 
circuits with multi-scroll attractors: application to communication," Communications in Nonlinear Science and Numerical Simulation, vol. 14, no. 6, pp. 2765-2775, 2009.

[14] F. Zhang, Y. Shu, and H. Yang, "Bounds for a new chaotic system and its application in chaos synchronization," Communications in Nonlinear Science and Numerical Simulation, vol. 16, no. 3, pp. 1501-1508, 2011.

[15] F. Zhang, X. Liao, and G. Zhang, "Some new results for the generalized Lorenz system," Qualitative Theory of Dynamical Systems, vol. 16, no. 3, pp. 749-759, 2017.

[16] C. Han, S. Yu, and G. Wang, "A Sinusoidally Driven Lorenz System and Circuit Implementation," Mathematical Problems in Engineering, vol. 2015, Article ID 706902, 2015.

[17] F. Zhang, X. Liao, G. Zhang, C. Mu, M. Xiao, and P. Zhou, "Dynamical behaviors of a generalized Lorenz family," Discrete and Continuous Dynamical Systems - Series B, vol. 22, no. 10, pp. 3707-3720, 2017.

[18] F. Zhang, C. Mu, P. Zheng, D. Lin, and G. Zhang, "The dynamical analysis of a new chaotic system and simulation," Mathematical Methods in the Applied Sciences, vol. 37, no. 12, pp. 1838-1846, 2014.

[19] G. A. Leonov and N. V. Kuznetsov, "Hidden attractors in dynamical systems: From hidden oscillations in hilbertkolmogorov, Aizerman, and Kalman problems to hidden chaotic attractor in chua circuits," International Journal of Bifurcation and Chaos, vol. 23, no. 1, Article ID 1330002, 2013.

[20] G. A. Leonov, N. V. Kuznetsov, and V. I. Vagaitsev, "Localization of hidden Chua's attractors," Physics Letters A, vol. 375, no. 23, pp. 2230-2233, 2011.

[21] G. A. Leonov, N. V. Kuznetsov, and T. N. Mokaev, "Hidden attractor and homoclinic orbit in Lorenz-like system describing convective fluid motion in rotating cavity," Communications in Nonlinear Science and Numerical Simulation, vol. 28, no. 1-3, pp. 166-174, 2015.

[22] B. Bao, F. Hu, M. Chen, Q. Xu, and Y. Yu, "Self-excited and hidden attractors found simultaneously in a modified chua's circuit," International Journal of Bifurcation and Chaos, vol. 25, no. 5, Article ID 1550075, 2015.

[23] S. Jafari and J. C. Sprott, "Simple chaotic flows with a line equilibrium," Chaos, Solitons \& Fractals, vol. 57, pp. 79-84, 2013.

[24] C. Li, J. C. Sprott, W. Thio, and H. Zhu, "A new piecewise linear hyperchaotic circuit," IEEE Transactions on Circuits and Systems II: Express Briefs, vol. 61, no. 12, pp. 977-981, 2014.

[25] P. R. Sharma, M. D. Shrimali, A. Prasad, N. V. Kuznetsov, and G. A. Leonov, "Controlling dynamics of hidden attractors," International Journal of Bifurcation and Chaos, vol. 25, no. 4, Article ID 1550061, 2015.

[26] Q. Li, H. Zeng, and X.-S. Yang, "On hidden twin attractors and bifurcation in the Chua's circuit," Nonlinear Dynamics, vol. 77, no. 1-2, pp. 255-266, 2014.

[27] B.-C. Bao, P. Jiang, Q. Xu, and M. Chen, "Hidden attractors in a practical Chua's circuit based on a modified Chua's diode," IEEE Electronics Letters, vol. 52, no. 1, pp. 23-25, 2016.

[28] B. C. Bao, Q. D. Li, N. Wang, and Q. Xu, "Multistability in Chua's circuit with two stable node-foci," Chaos: An Interdisciplinary Journal of Nonlinear Science, vol. 26, no. 4, Article ID 043111, 2016.

[29] J. C. Sprott, X. Wang, and G. Chen, "Coexistence of point, periodic and strange attractors," International Journal of Bifurcation and Chaos, vol. 23, no. 5, Article ID 1350093, 2013.
[30] B. C. Bao, H. Bao, N. Wang, M. Chen, and Q. Xu, "Hidden extreme multistability in memristive hyperchaotic system," Chaos, Solitons \& Fractals, vol. 94, pp. 102-111, 2017.

[31] H. Bao, N. Wang, B. Bao, M. Chen, P. Jin, and G. Wang, "Initial condition-dependent dynamics and transient period in memristor-based hypogenetic jerk system with four line equilibria," Communications in Nonlinear Science and Numerical Simulation, vol. 57, pp. 264-275, 2018.

[32] J. Kengne, Z. N. Tabekoueng, V. K. Tamba, and A. N. Negou, "Periodicity, chaos, and multiple attractors in a memristorbased Shinriki's circuit," Chaos: An Interdisciplinary Journal of Nonlinear Science, vol. 25, no. 10, Article ID 103126, 2015.

[33] C. Li and J. C. Sprott, "Multistability in the lorenz system: A broken butterfly," International Journal of Bifurcation and Chaos, vol. 24, no. 10, Article ID 1450131, 2014.

[34] Q. Xu, Y. Lin, B. Bao, and M. Chen, "Multiple attractors in a nonideal active voltage-controlled memristor based Chua's circuit," Chaos, Solitons \& Fractals, vol. 83, pp. 186-200, 2016.

[35] Q. Xu, Q. Zhang, N. Wang, H. Wu, and B. Bao, "An improved memristive diode bridge-based band pass filter chaotic circuit," Mathematical Problems in Engineering, vol. 2017, Article ID 2461964, 11 pages, 2017. 


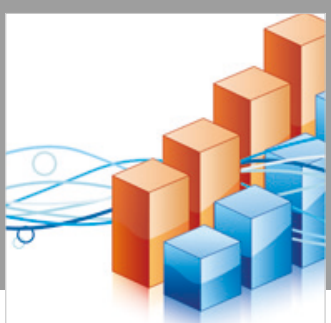

Advances in

Operations Research

\section{-n-m}
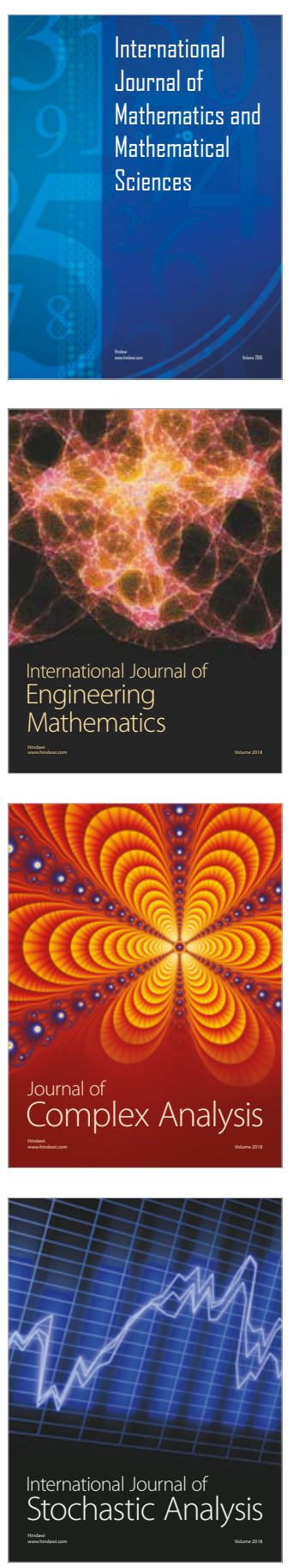
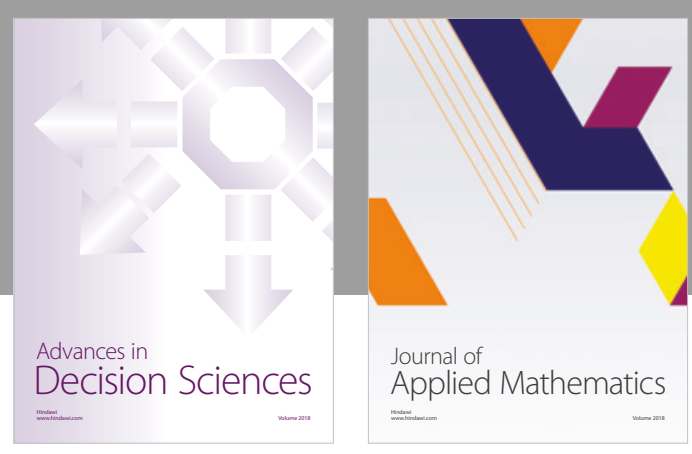

Journal of

Applied Mathematics
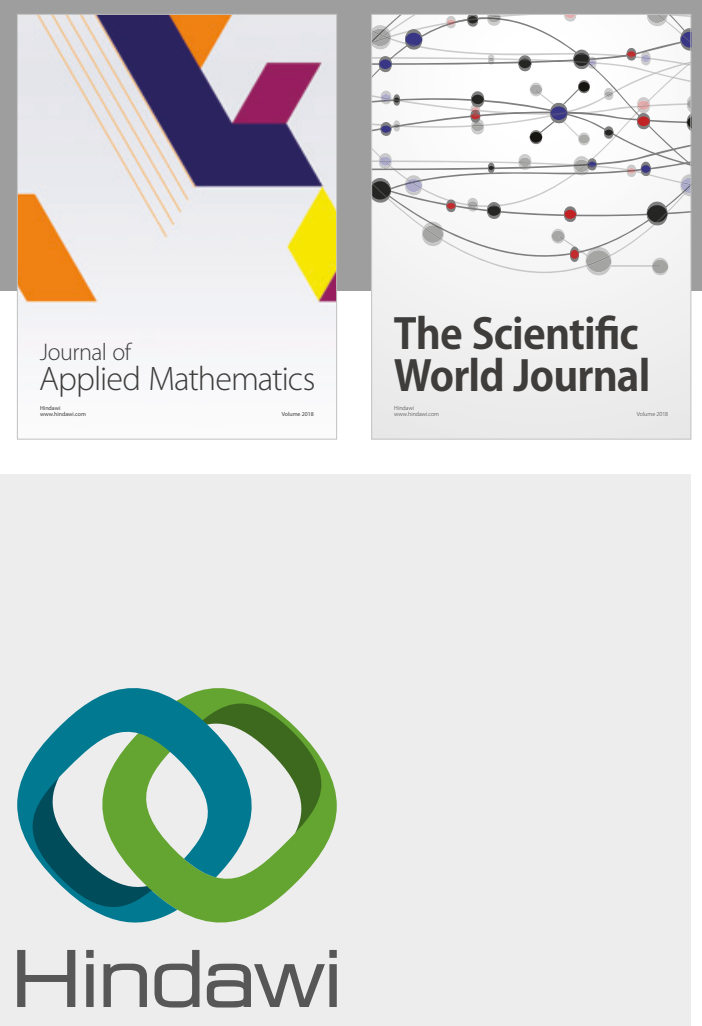

Submit your manuscripts at

www.hindawi.com

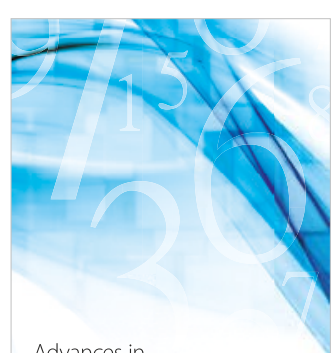

Advances in
Numerical Analysis
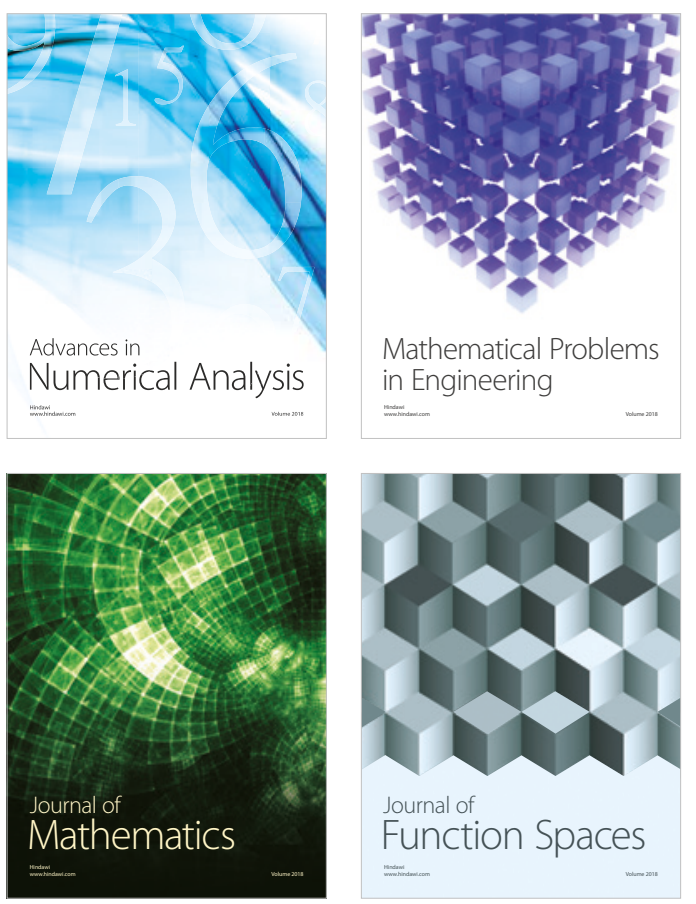

Mathematical Problems in Engineering

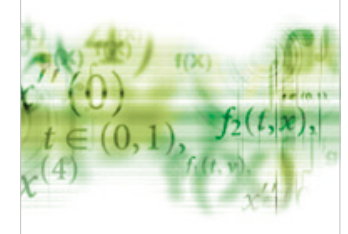

International Journal of

Differential Equations

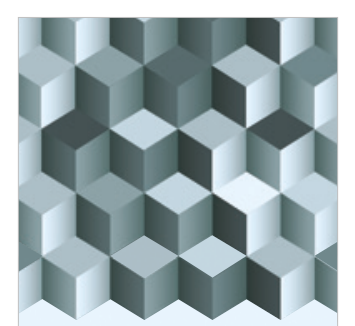

Journal of

Function Spaces

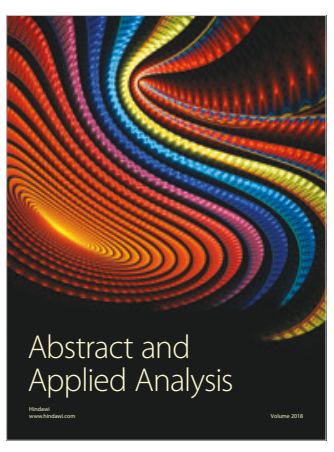

The Scientific

World Journal

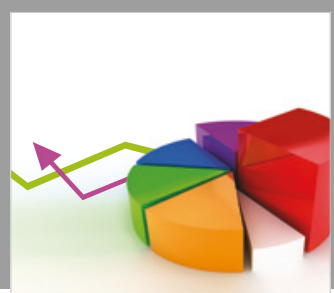

Journal of

Probability and Statistics
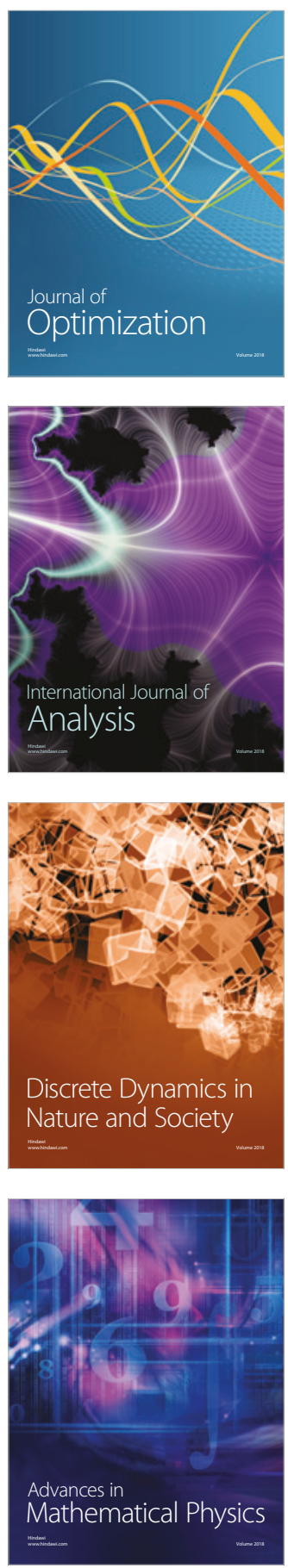